\title{
Hepatic Hodgkin Lymphoma Presenting as Solitary Hepatic Mass Following Other latrogenic Immunodeficiency- Associated Lymphoproliferative Disorder in a Patient With Rheumatoid Arthritis
}

\author{
Yasuhiro Tanaka ${ }^{\mathrm{a}, \mathrm{b}, \mathrm{d}}$, Satsuki Asai ${ }^{\mathrm{c}}$, Akiko Hashimoto $^{\mathrm{a}}$, Isaku Shinzato ${ }^{\mathrm{a}}$
}

\begin{abstract}
Lymphoproliferative disorders (LPDs) occur frequently in patients with rheumatoid arthritis (RA) under methotrexate treatment. Some LPDs spontaneously regressed after methotrexate discontinuation, but classic Hodgkin lymphoma (CHL)-type LPDs frequently relapse, and chemotherapy is usually required for the treatment. CHL usually spreads in contiguous lymph nodes and then infiltrates in organs at an advanced stage. Thus, hepatic Hodgkin lymphoma (HHL) without lymphadenopathy is extremely rare at diagnosis. We present a case of methotrexate-associated LPDs associated with systemic lymphadenopathy and hepatosplenic mass in a 71-year-old woman with RA under methotrexate treatment over 10 years. Although spontaneous remission occurred after methotrexate discontinuation, she developed HHL presenting as a solitary hepatic mass without lymphadenopathy 3 years after spontaneous regression. She received brentuximab vedotin (BV) combination chemotherapy without bleomycin to avoid pulmonary toxicity. Complete metabolic response was achieved after four courses of BV combination chemotherapy, and the activity of RA was kept to be in remission. Our case suggested that the recurrence lesions of LPDs may present at unexpected site, which is not coincide with the primary site, and BV combination chemotherapy is a promising regimen for limitedstage CHL-type LPDs in patients with RA owing to its anti-lymphoma effect on CHL-type LPDs and a possible targeted therapy for RA.
\end{abstract}

Keywords: Classic Hodgkin lymphoma; Lymphoproliferative disorders; Hepatic mass; Methotrexate; Brentuximab vedotin; Combination chemotherapy

Manuscript submitted December 15, 2020, accepted December 23, 2020 Published online December 30, 2020

aDepartment of Hematology and Clinical Immunology, Kobe City Nishi-Kobe Medical Center, 5-7-1, Koji-dai, Nishi-ku, Kobe City, 651-2273 Hyogo, Japan ${ }^{b}$ Division of Hematology, Japanese Red Cross Society Wakayama Medical Center, 4-20, Komatsubaradori, Wakayama City, 640-8558 Wakayama, Japan 'Department of Pathology, Kobe City Nishi-Kobe Medical Center, 5-7-1, Koji-dai, Nishi-ku, Kobe City, 651-2273 Hyogo, Japan

${ }^{\mathrm{d} C}$ Corresponding Author: Yasuhiro Tanaka, Division of Hematology, Japanese Red Cross Society Wakayama Medical Center, 4-20, Komatsubaradori, Wakayama City, 640-8558 Wakayama, Japan.

Email: yasutanaka1717@gmail.com

doi: https://doi.org/10.14740/jmc3636

\section{Introduction}

Classic Hodgkin lymphoma (CHL) is a lymphoid hematological malignancy characterized by clinically lymph node enlargement and pathologically characteristic appearance of Hodgkin cells and Reed-Sternberg cells. It usually spreads in contiguous lymph nodes along the lymphatic system and then infiltrates in organs such as the liver, bone, and lung [1]. Thus, hepatic involvement usually occurs late during the disease or at an advanced stage, and hepatic presentation without lymphadenopathy is extremely rare [2]. The standard treatment regimen consists of doxorubicin, bleomycin, vinblastine, and dacarbazine (ABVD), but pulmonary sequelae due to bleomycin toxicity usually occur after ABVD chemotherapy. Brentuximab vedotin (BV) is monoclonal anti-CD30 antibody conjugated with monomethyl auristatin E. Hodgkin cells and Reed-Sternberg cells usually express CD30, and CD30 is the target antigen for $\mathrm{BV}$. BV in combination with $\mathrm{ABVD}$ chemotherapy without bleomycin has recently been reported to show favorable efficacy for CHL [3]. We report here a case of hepatic Hodgkin lymphoma presenting as a solitary hepatic mass following other iatrogenic immunodeficiency-associated lymphoproliferative disorders (OII-LPDs) in a patient with rheumatoid arthritis (RA). Our patient received BV combination chemotherapy and achieved complete response. This is the first case of hepatic Hodgkin lymphoma in which complete metabolic response was achieved with $\mathrm{BV}$ combination chemotherapy in a patient with RA.

\section{Case Report}

A 71-year-old woman was admitted to our hospital for the evaluation of multiple hepatic masses in December 2015. She was diagnosed as having RA in 1992 at another hospital and had been taking methotrexate for RA for over 10 years. She had a fever of more than $38{ }^{\circ} \mathrm{C}$ for over 2 months, and her laboratory examination showed an elevated level of C-reactive protein (CRP). Computed tomography (CT) showed multiple lymphadenopathies in bilateral supraclavicular and mediastinal regions, and multiple masses in the liver and spleen. Methotrexate was discontinued before her admission to our hospi- 
tal, and positron emission tomography computed tomography (PET-CT) showed no accumulation of fluorodeoxyglucose in the whole body after methotrexate discontinuation. Thus, we suspected OII-LPDs, especially methotrexate-associated LPD (MTX-LPD), although a histological diagnosis of OIILPD was not obtained owing to spontaneous regression. After methotrexate discontinuation, polyarthritis appeared, and etanercept was started for RA in December 2017. However, CT showed a solitary hepatic mass in October 2018. After etanercept was switched to abatacept, the hepatic mass continued to grow. She was readmitted to our hospital after abatacept discontinuation in September 2019. Physical examination revealed no apparent abnormalities of the thorax and abdomen. The hepatic mass was not palpable, and ulnar drift deformities were found in all fingers, but no joint swelling was found. Laboratory examination revealed mild anemia (hemoglobin level: $11.1 \mathrm{~g} / \mathrm{dL}$ ) and increased levels of alkaline phosphatase (ALP) (547 IU/L, normal range: 165 - 320), soluble interleukin (IL)-2 receptor (720 IU/L, normal range: 121 - 613) and matrix metalloproteinase-3 $(258.5 \mathrm{ng} / \mathrm{mL}$, normal range: 17.3 - 59.7). The levels of CRP and lactic dehydrogenase were within the normal range. The titers of the rheumatoid factor and anti-cyclic citrullinated peptide antibody increased to 225 $\mathrm{IU} / \mathrm{mL}$ (normally below 18) and $273 \mathrm{U} / \mathrm{ml}$ (normally below 4.5), respectively. CT showed a solitary mass in the right lobe of the liver (Fig. 1a). Magnetic resonance imaging (MRI) showed a low-intensity solitary mass on T1-weighted images (Fig. 1b) and a high-intensity solitary mass on T2-weighted (Fig. 1c) and diffusion-weighted images in the anterior segment of the right lobe (Fig. 1d). PET-CT showed abnormal accumulation of fluorodeoxyglucose in the solitary hepatic mass (Fig. 1e). Thus, liver biopsy was performed in October 2019. Histopathological examination showed that large-sized abnormal cells and dense inflammatory cells were found in a biopsied liver sample (Fig. 2a). Among the dense inflammatory cells, most were CD3-positive T lymphocytes (Fig. 2b) and a few were CD20-positive B lymphocytes. Immunohistochemical examination showed that the large abnormal cells were positive for CD30 (Fig. 2c) and MUM-1, and negative for CD3, CD5, CD10, CD15, CD20, and CD79. The EpsteinBarr virus-encoded small RNAs (EBERs) in situ hybridization was positive for abnormal cells (Fig. 2d). The Ki-67 labelling index was high (Fig. 2e). These findings led to the diagnosis of hepatic Hodgkin lymphoma-type OII-LPD in accordance with the World Health Organization (WHO) 2016 classification [4]. Chromosomal analysis using $\mathrm{G}$ banding was not carried out. Bone marrow examination showed no abnormal cells.

She was not treated with methotrexate because of her history of probable MTX-LPD. Biologics have been used for the treatment of RA, but they pose the risk of infections, especially pneumonia, and bleomycin has been reported to have pulmonary toxicity. Thus, we selected BV instead of bleomycin. BV combination AVD (BV, $50 \mathrm{mg}$ per body; doxorubicin, $30 \mathrm{mg}$ per body; vinblastine, $7.5 \mathrm{mg}$ per body; dacarbazine, $460 \mathrm{mg}$ per body) chemotherapy was administered every 2 weeks. She did not complain of any adverse effects. After two courses of BV combination chemotherapy, interim PET-CT showed no abnormal accumulation of fluorodeoxyglucose in the whole body including systemic large joints. She received four cours- es of BV combination chemotherapy; as a result, she achieved and maintained complete metabolic response (CMR) as shown by PET-CT (Fig. 1f). She has been alive in CMR with no flareup of RA without any medical treatment over 1 year.

\section{Discussion}

Here, we report a rare case of hepatic Hodgkin lymphoma following OII-LPD in a patient with RA. The standardized incidence ratio of LPDs is increased in RA patients treated with methotrexate $[4,5]$. MTX-LPDs are complications of methotrexate treatment in patients with RA. The most frequent histological subtype of MTX-LPDs is diffuse large B-cell lymphoma followed by CHL. Momose et al [6] reported that CHL-type OII-LPDs (CHL-LPDs) account for about 13\% of OII-LPDs, among which many showed involvement of lymph nodes or a combination of lymph nodes and other organs, such as the liver and lung. However, to the best of our knowledge, only one case of OII-LPD that involved solely the liver has been reported. Tsukazaki et al [7] reported the case of an 88-year-old female with RA who developed hepatosplenic Hodgkin lymphoma. She had been taking methotrexate and infliximab for 6 years, and she developed lymph node enlargement and hepatosplenomegaly. Hodgkin lymphoma was diagnosed on the basis of the finding of cervical lymph node biopsy. Spontaneous regression occurred after the withdrawal of methotrexate and infliximab, but after 1 year, her Hodgkin lymphoma relapsed presenting as hepatosplenomegaly without lymphadenopathy. She was treated with four cycles of ABVD chemotherapy and achieved a complete response. In our patient, multiple hepatosplenic mass and lymph nodes enlargement were found on CT scans when she was under methotrexate treatment; and about 3 years later, a solitary haptic mass without lymphadenopathy was found on PET-CT scans when she was under the treatment of biologics such as etanercept and abatacept. She was treated with BV combination chemotherapy and achieved CMR, as shown by PET-CT. This is the second case of a patient with RA with a hepatic presentation of classic Hodgkin lymphoma recurring following OII-LPD.

Patients with primary hepatic lymphoma (PHL) presented with hepatic failure or encephalopathy. Imaging methods such as CT showed hepatomegaly without any mass [8]. This indicated a diffuse infiltration pattern, as in the case reported by Tsukazaki et al. Almost all cases with the diffuse infiltration pattern of PHL showed secondary hepatic involvement in an advanced stage of CHL. Otherwise, patients with PHL presented with no obvious constitutional symptoms such as fever, weight loss, and general malaise. Imaging methods showed a solitary mass or multiple masses in the liver without lymphadenopathy [2]. This indicated the nodular infiltration pattern, as in our case. In the case of primary non-Hodgkin lymphoma of the liver, Emile et al [9] showed that the diffuse infiltration pattern indicated a worse prognosis than the nodular infiltration pattern. However, in the case of CHL, there has been no report about whether the diffuse infiltration pattern has a worse prognosis than the nodular infiltration pattern. More cases should be collected to clarify this point. 

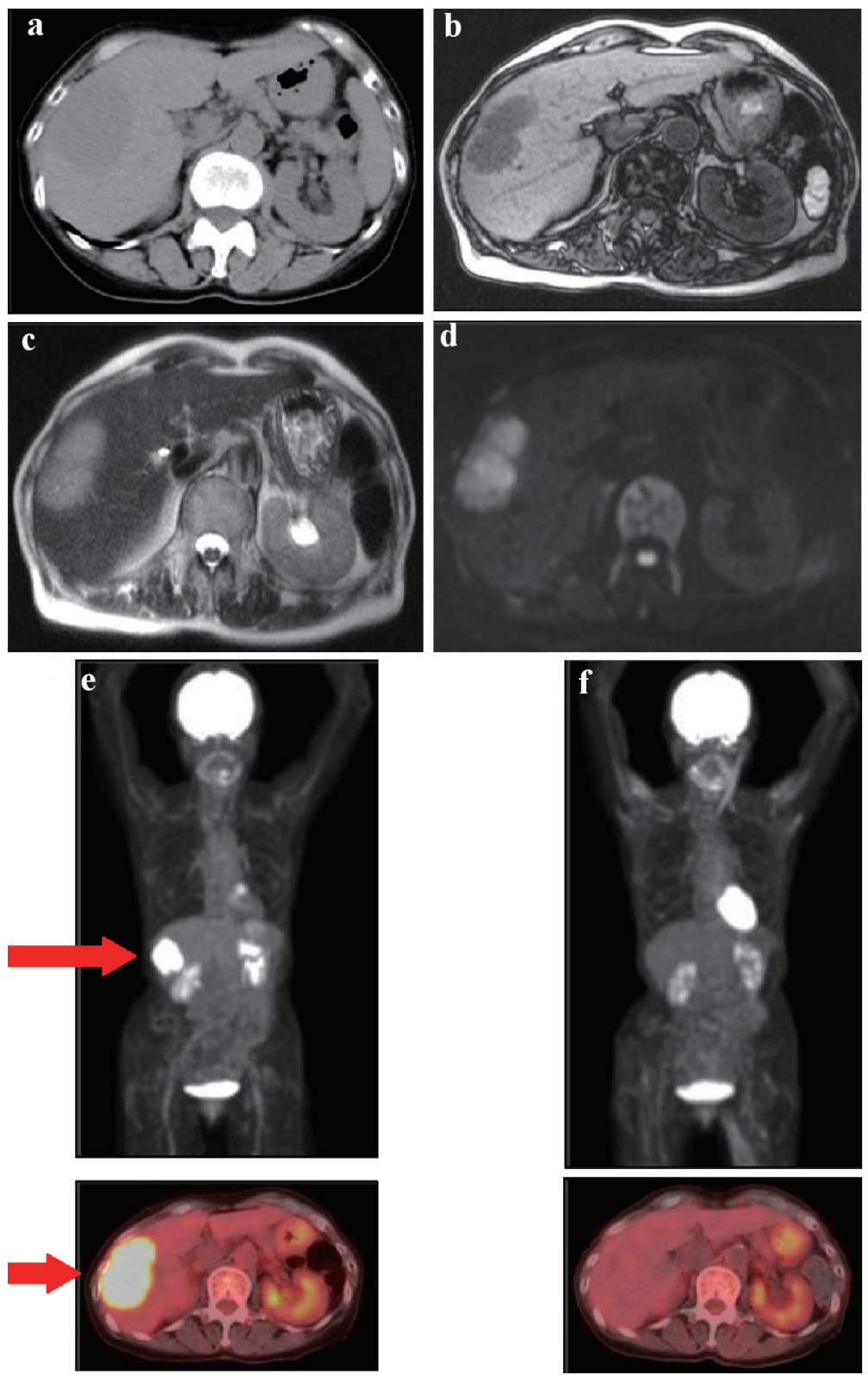

Figure 1. Images of hepatic mass. (a) CT images showed a solitary low-density mass in the right lobe of the liver. MRI showed a low-intensity solitary hepatic mass on T1-weighted image (b), a high-intensity mass on T2-weighted image (c), and a highintensity mass on diffusion-weighted image (d). (e) PET-CT images showed abnormal accumulation of fluorodeoxyglucose in liver (arrow); (upper panel) whole-body PET-CT image, (lower panel) transverse section of PET-CT image. (f) PET-CT images after four courses of BV combination chemotherapy showed no abnormal accumulation of fluorodeoxyglucose in whole body, which indicated that she remained in complete metabolic remission; (upper panel) whole-body PET-CT image, (lower panel) transverse section of PET-CT image. CT: computed tomography; MRI: magnetic resonance imaging; PET-CT: positron emission tomography computed tomography.

Patients with RA frequently have pulmonary complications including interstitial pneumonia and are treated with immunosuppressive drugs such as methotrexate or tacrolimus, prednisolone, and biologics. These drugs lead to immunosuppression and complications, especially infectious pneumonia [10]. On the other hand, bleomycin leads to pulmonary fibrosis and fatal pulmonary complications in patients with CHL $[11,12]$. Thus, extra care should be taken for patients with RA and CHL for pulmonary complications under treatment with ABVD chemotherapy. Although ABVD chemotherapy is the standard treatment for CHL, Abramson et al [13] reported that BV combination chemotherapy without consolidation radiotherapy is effective for nonbulky limited-stage CHL in a multicenter phase 2 study. Thirty-four patients were enrolled, and those who received four cycles of $\mathrm{BV}$ combination chemotherapy achieved a high complete response rate. Ichikawa et al 

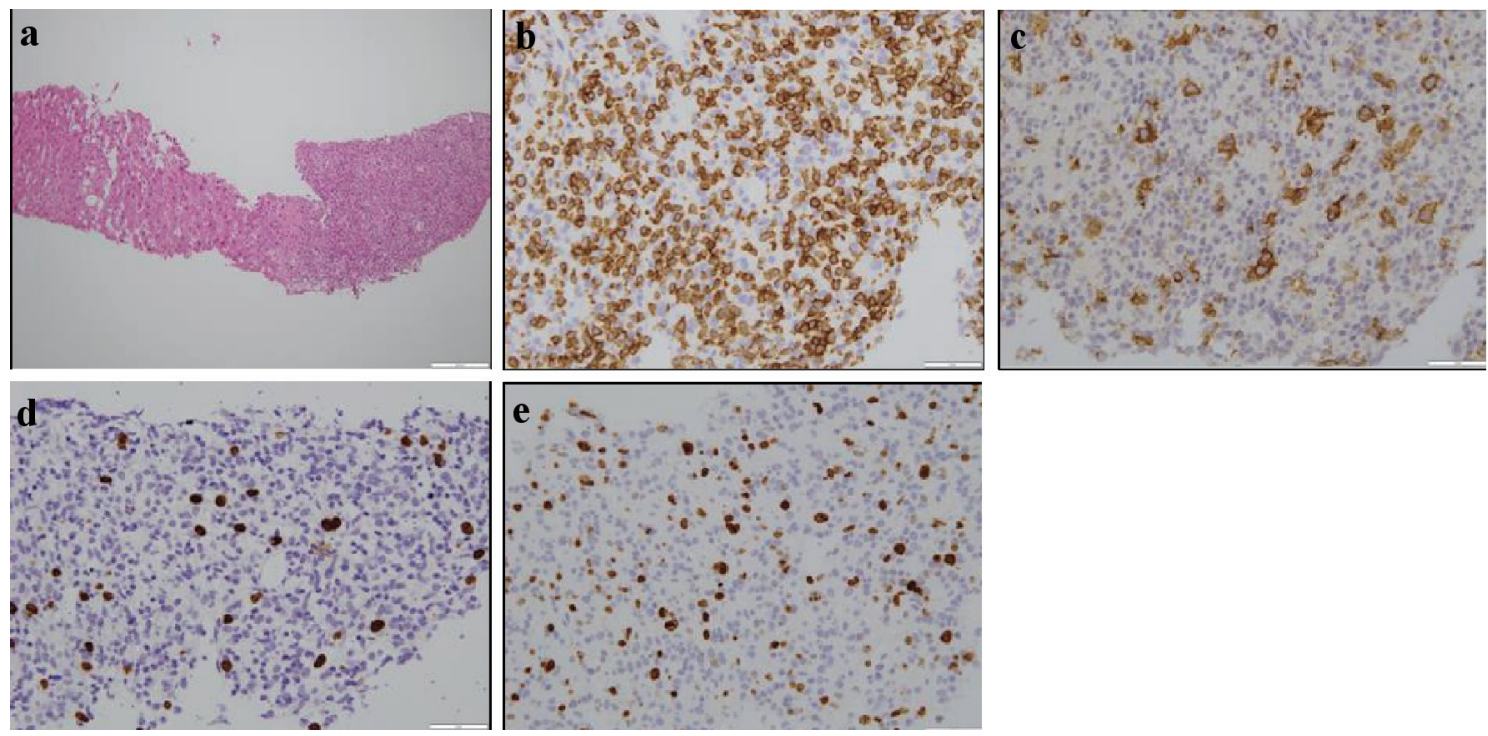

Figure 2. Histopathological findings of biopsied liver sample. (a) Diffuse infiltration of many cells was observed on the right side of the biopsied liver sample. Normal hepatocytes were observed on the left side (hematoxylin and eosin staining; original magnification, $\times 10)$. (b) Immunohistochemical analysis showed that small inflammatory cells were positive for CD3 (original magnification, $\times 400$ ). (c) Immunohistochemical analysis showed that abnormal cells were positive for CD30 (original magnification, $\times 400)$. (d) Epstein-Barr virus-encoded small RNAs (EBERs) in situ hybridization was positive for abnormal cells (original magnification, $x$ 400). (e) The Ki-67 index was high (original magnification, $\times 400$ ).

[14] reported that three patients with methotrexate-associated advanced-stage CHL received six courses of BV combination chemotherapy and achieved CMR without pulmonary toxicity. Our patient received four courses of BV combination chemotherapy and achieved CMR as shown by PET-CT. Thus, this is the first case in which BV combination chemotherapy was effective for limited-stage hepatic CHL-LPD in a patient with RA. Recently, Vachhani et al [15] and Nakazato et al [16] have reported that BV may represent a novel targeted therapy for RA. This point may support the choice of BV combination chemotherapy instead of ABVD chemotherapy for CHL-LPDs in patients with RA.

In conclusion, we report a case of hepatic Hodgkin lymphoma presenting as a solitary hepatic mass following OIILPD in a patient with RA. Our patient achieved CMR after four courses of BV combination chemotherapy. Our case suggested that the recurrence lesions of LPDs may present at unexpected site, which is not coincide with the primary site. BV combination chemotherapy is a promising regimen for CHLLPDs in patients with RA owing to its anti-lymphoma effect on CHL-LPDs and a possible targeted therapy for RA.

\section{Acknowledgments}

We would like to thank for the medical staff at the Nishi-Kobe Medical Center who were involved in the care of this patient.

\section{Financial Disclosure}

All authors have no financial disclosure to declare.

\section{Conflict of Interest}

All authors declare no conflict of interest.

\section{Informed Consent}

We verbally obtained the informed consent from the patient for the publication of this case report and accompanying images.

\section{Author Contributions}

YT designed the study, collected the data and wrote the paper; SA provided all the pathological pictures; $\mathrm{AH}$ and IS treated this patient; IS supervised this study. All authors contributed to the editing of this manuscript.

\section{Data Availability}

The authors declare that data supporting the findings of this study are available within the article.

\section{References}

1. Musshoff K. Prognostic and therapeutic implications of staging in extranodal Hodgkin's disease. Cancer Res. 1971;31(11):1814-1827.

2. Chim CS, Choy C, Ooi CG, Liang R. Hodgkin's disease 
with primary manifestation in the liver. Leuk Lymphoma. 2000;37(5-6):629-632.

3. Connors JM, Jurczak W, Straus DJ, Ansell SM, Kim WS, Gallamini A, Younes A, et al. Brentuximab Vedotin with chemotherapy for Stage III or IV Hodgkin's Lymphoma. N Engl J Med. 2018;378(4):331-344.

4. Galard P, Swerdlow SH, Harris NL, Sundstrom C, Jaffe ES. Other iatrogenic immunodeficiency-associated lymphoproliferative disorders. In: Swerdlow SH, Campo E, Harris NL, Jaffe ES, Pileri SA, Stein H, Thiele J, et al. eds. WHO classification of tumors of haematopoietic and lymphoid tissues. Revised 4th ed, Lyon: IARC, 2017: p. 462-464.

5. Gion Y, Iwaki N, Takata K, Takeuchi M, Nishida K, Orita Y, Tachibana T, et al. Clinicopathological analysis of methotrexate-associated lymphoproliferative disorders: Comparison of diffuse large B-cell lymphoma and classical Hodgkin lymphoma types. Cancer Sci. 2017;108(6):12711280 .

6. Momose S, Tamaru JI. Iatrogenic immunodeficiency-associated lymphoproliferative disorders of B-cell type that develop in patients receiving immunosuppressive drugs other than in the post-transplant setting. J Clin Exp Hematop. 2019;59(2):48-55.

7. Tsukazaki Y, Shinohara T, Tanaka K, Naruse K, Iwahara Y, Inoue S. Hepatosplenic Hodgkin lymphoma without lymphadenopathy following reversible methotrexate-associated lymphoproliferative disorder. Mod Rheumatol. 2017;27(2):372-375.

8. Lefkowitch JH, Falkow S, Whitlock RT. Hepatic Hodgkin's disease simulating cholestatic hepatitis with liver failure. Arch Pathol Lab Med. 1985;109(5):424-426.

9. Emile JF, Azoulay D, Gornet JM, Lopes G, Delvart V,
Samuel D, Reynes M, et al. Primary non-Hodgkin's lymphomas of the liver with nodular and diffuse infiltration patterns have different prognoses. Ann Oncol. 2001;12(7):1005-1010.

10. Spagnolo P, Lee JS, Sverzellati N, Rossi G, Cottin V. The lung in rheumatoid arthritis: focus on interstitial lung disease. Arthritis Rheumatol. 2018;70(10):1544-1554.

11. Martin WG, Ristow KM, Habermann TM, Colgan JP, Witzig TE, Ansell SM. Bleomycin pulmonary toxicity has a negative impact on the outcome of patients with Hodgkin's lymphoma. J Clin Oncol. 2005;23(30):7614-7620.

12. Sun HL, Atenafu EG, Tsang R, Kukreti V, Marras TK, Crump M, Kuruvilla J. Bleomycin pulmonary toxicity does not adversely affect the outcome of patients with Hodgkin lymphoma. Leuk Lymphoma. 2017;58(11):2607-2614.

13. Abramson JS, Arnason JE, LaCasce AS, Redd R, Barnes JA, Sokol L, Joyce R, et al. Brentuximab vedotin, doxorubicin, vinblastine, and dacarbazine for nonbulky limited-stage classical Hodgkin lymphoma. Blood. 2019;134(7):606-613.

14. Ichikawa S, Fukuhara N, Saito K, Onodera K, Shirai T, Onishi Y, Yokoyama H, et al. Successful treatment of methotrexate-associated classical Hodgkin lymphoma with brentuximab vedotin-combined chemotherapy: a case series. Int J Hematol. 2020;111(5):667-672.

15. Vachhani P, Bose N, Brodeur JP, Holkova B, Bose P. Remission of rheumatoid arthritis on brentuximab vedotin. Rheumatology (Oxford). 2014;53(12):2314-2315.

16. Nakazato T, Takanashi S, Hirano M, Ito C, Fujita Y, Osada Y, Aisa Y, et al. Brentuximab vedotin is effective for rheumatoid arthritis in a patient with relapsed methotrexate-associated Hodgkin lymphoma. Ann Hematol. 2018;97(8):1489-1491. 